\title{
EINE KENNZEICHNUNG SEMI-PERFEKTER MODULN
}

\author{
FR. KASCH und E. A. MARES
}

Dem Gedenken an TAdasi NaKayama gewidmet

1. Ein projektiver Modul wird (in [1]) semi-perfekt genannt, wenn jedes epimorphe Bild von ihm eine projektive Hülle besitzt. Eine projektive Hülle eines Moduls $C$ ist eine exakte Fole $P \stackrel{f}{\rightarrow} C \longrightarrow 0$, wobei $P$ projektiv ist und der Kern $K e(f)$ von $f$ Klein ( = small = superflous $)^{*}$ in $P$ ist. In [1] wird gezeigt, daß ein projektiver Modul $P$ dann und nur dann semi-perfekt ist, wenn das Radikal $R a(P)$ von $P$ klein in $P$ ist, $\bar{P}=P / R a(P)$ halbeinfach ist und jede direkte Zerlegung von $\bar{P}$ durch eine direkte Zerlegung von $P$ induziert wird.

Dieses Resultat soll hier benutzt werden, um eine weitere Charakterisierung semi-perfekter Moduln zu beweisen, die ebenso einfach wie bemerkenswert ist und darüber hinaus durch Dualisierung eine einfache Konstruktion der injektiven Hülle liefert.

2. Es sei $R$ ein Ring mit 1-Element und alle Moduln seien unitäre $R$ Rechtsmoduln. Ein Modul $M$ heiße komplementiert (bezüglich der Addition), wenn $z u$ jedem Untermodul $U \subset M$ in der Menge der Untermoduln $V \subset M$ mit $M=U+V$ (mindestens) ein minimaler existiert ; jeder solche minimale Untermodul heiße ein Komplement von $U$ und eines dieser Komplemente werde mit $U^{\prime}$ bezeichnet.

Hilfssatz. Ist $U^{\prime}$ ein Komplement von $U$ in $M$, dann ist $U \cap U^{\prime}$ klein in $U^{\prime}$. Ist $U^{\prime \prime}$ ein Komplement von $U^{\prime}$ in $M$, dann ist $U^{\prime \prime} \cap U^{\prime}$ klein in $U^{\prime}$.

Beweis. Sei $U \cap U^{\prime}+B=U^{\prime}$ mit $B \subset U^{\prime}$, dann folgt $M=U+U \cap U^{\prime}+B=U+B$ und wegen der Minimalität von $U^{\prime}$ folgt $B=U^{\prime}$, d.h. $U \cap U^{\prime}$ ist klein in $U^{\prime}$. Sei $U^{\prime \prime} \cap U^{\prime}+B=U^{\prime}$ mit $B \subset U^{\prime}$, dann folgt $M=U+U^{\prime \prime} \cap U^{\prime}+B$; da nach der schon bewiesenen ersten Aussage des Hilfssatzes $U^{\prime \prime} \cap U^{\prime}$ klein in $U^{\prime \prime}$ ist, ist

Received May 17, 1965.

* Ein Untermodul $K$ von $P$ heisst klein in $P$, wenn für jeden Untermodul $U=P, U \neq P$ auch $U+K \neq P$. 
$U^{\prime \prime} \cap U^{\prime}$ auch klein in $M$, also folgt $M=U+B$ und daraus ergibt sich wieder $B=U^{\prime}$.

3. Wir kommen nun zu der angekündigten Kennzeichnung semi-perfekter Moduln.

SATz. Ein projektiver Modul ist dann und nur dann semi-perfekt, wenn er komplementiert ist.

Beweis.

"Nur dann". Sei $P$ ein semi-perfekter Modul und $U \subset P$ ein Untermodul. Bezeichne $\bar{U}$ das Bild von $U$ in $\bar{P}=P / R a(P)$ bei dem natürlichen Epimorphismus $\stackrel{\stackrel{\nu}{\longrightarrow}}{\longrightarrow} \bar{P}$. Da $\bar{P}$ halbeinfach ist, existiert ein Untermodul $\Lambda \subset \bar{P}$ mit $\bar{P}=\bar{U} \oplus \Lambda$. Nach Voraussetzung existiert eine Zerlegung $P=U_{1} \oplus V$ mit $\bar{U}_{1}=\bar{U}, \bar{V}=\Lambda$. Aus $\bar{U}_{1}=\bar{U}$ folgt $U_{1}+R a(P)=U+R a(P)$ und daher gilt $P=U+R a(P)+V$. Da $R a(P)$ klein in $P$ ist, folgt $P=U+V$. Angenommen $P=U+V_{0}$ mit $V_{0} \subset V$, dann folgt $\bar{V}_{0} \subset \bar{V}=\Lambda$ und $\bar{P}=\bar{U}+\bar{V}_{0}=\bar{U} \oplus \bar{V}_{0}=\bar{U} \oplus \bar{V}$, also $\bar{V}_{0}=\bar{V}=\Lambda$. Daraus folgt $V_{0}+R a(P)=V+R a(P)$ und da $R a(P)$ klein in $P$ ist und $V_{0} \subset V$ ergibt sich

$$
P=U_{1} \oplus V=U_{1}+V_{0}+R a(P)=U_{1}+V_{0}=U_{1} \oplus V_{0},
$$

also $V_{0}=V$, d.h. $V$ ist ein Komplement von $U$.

"Dann". Sei $P$ projektiv und komplementiert und sei $P \stackrel{f}{\longrightarrow} C \longrightarrow 0$ exakt. Wir können und wollen voraussetzen, daß $C=P / U$ und $f=\nu$ der natürliche Epimorphismus von $P$ auf den Faktormodul $P / U$ sind. Sei $P=U+U^{\prime}$ mit einem Komplement $U^{\prime}$ von $U$. Wegen $P / U=\left(U+U^{\prime}\right) / U$ folgt dann, daß $\nu^{\prime}=$ $\nu \mid U^{\prime}$ ( = Einschränkung von $\nu$ auf $U^{\prime}$ ) ein Epimorphismus von $U^{\prime}$ auf $P / U$ ist, dessen Kern $K e\left(\nu^{\prime}\right)=U \cap U^{\prime}$ nach dem Hilfssatz klein in $U^{\prime}$ ist.

Bisher haben wir nicht benutzt, daß $P$ projektiv ist. Mit dieser Voraussetzung kann nun gezeigt werden, daß $U^{\prime}$ direkter Summand von $P$ und daher selbst wieder projektiv ist. Dann ist

$$
U^{\prime} \stackrel{\nu^{\prime}}{\longrightarrow} P / U \longrightarrow 0
$$

offenbar eine projektive Hülle von $P / U$ und der Beweis ist vollständig. Sei $P=U^{\prime \prime}+U^{\prime}$ mit einem Komplement $U^{\prime \prime}$ von $U^{\prime}$. Seien

$$
\mu: P \rightarrow P / U^{\prime \prime} \cap U^{\prime}, \quad \mu^{\prime}=\mu \mid U^{\prime}
$$


und sei $\pi$ die Projektion von $P / U^{\prime \prime} \cap U^{\prime}=U^{\prime \prime} / U^{\prime \prime} \cap U^{\prime} \oplus U^{\prime} / U^{\prime \prime} \cap U^{\prime}$ auf $U^{\prime} / U^{\prime \prime} \cap U^{\prime}$. Dann existiert wegen der Projektivität von $P$ ein Homomorphismus $f$ so, daB das Diagramm

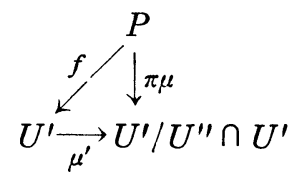

kommutativ ist. Aus $\pi \mu=\mu^{\prime} f$ folgt

$$
\pi \mu\left(U^{\prime}\right)=U^{\prime} / U^{\prime \prime} \cap U^{\prime}=\mu^{\prime} f\left(U^{\prime}\right)
$$

und daher gilt $f\left(U^{\prime}\right)+K e\left(\mu^{\prime}\right)=U^{\prime} . \quad$ Da $K e\left(\mu^{\prime}\right)=U^{\prime \prime} \cap U^{\prime}$ nach dem Hilfssatz klein in $U^{\prime}$ ist, folgt $f\left(U^{\prime}\right)=U^{\prime}$ und folglich gilt $P=K e(f)+U^{\prime}$. Wegen $K e(f) \subset K e(\pi \mu)=U^{\prime \prime}$ und wegen der Minimalität von $U^{\prime \prime}$ folgt $K e(f)=U^{\prime \prime}$. Andererseits gilt

$$
\left.U^{\prime \prime}=K e(\pi \mu)=K e^{\prime} \mu^{\prime} f\right)=f^{-1}\left(K e\left(\mu^{\prime}\right)\right)=f^{-1}\left(U^{\prime \prime} \cap U^{\prime}\right)
$$

und da $f$ ein Epimorphismus ist, folgt $0=f\left(U^{\prime \prime}\right)=U^{\prime \prime} \cap U^{\prime}$, was zu zeigen war.

4. Bemerkungen und Folgerungen.

a) Der zweite Teil des Beweises kann so formuliert werden, daß für $P$ nicht Projektivität sondern nur Selbstprojektivität vorausgesetzt wird. Dazu betrachte man bei sonst gleichen Bezeichnungen wie zuvor das kommutative Diagramm

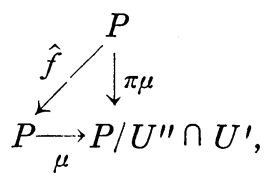

wobei $\hat{f}$ nach Voraussetzung existiert. Dann gilt $B i(\hat{f}) \subset U^{\prime}$ und man erhält die Abbildung $f$ im Beweis des Satzes aus $\hat{f}$ durch Einschränkung des Zieles $P$ von $\hat{f}$ auf $U^{\prime}$.

\section{b) Existenz der injektiven Hülle}

Sei $U \subset Q$, dann gibt es in der Menge der Untermoduln $V \subset Q$ mit $U \cap V=0$ nach dem Zornschen Lemma (mindestens) einen maximalen, der hier ein Komplement genannt und mit $U^{*}$ bezeichnet werden soll. Ebenso gibt es ein Komplement $U^{* *}$ von $U^{*}$ mit $U \subset U^{* *}$. Der zum Begriff "klein" duale 
Begriff ist "groß"( = large = essential $)$. Damit kann der zweite Teil des Beweises dualisiert werden und liefert einen neuen Beweis für die Existenz der injektiven Hülle, bei dem das Zornsche Lemma nur zum Existenzbeweis von $U^{*}$ und $U^{* *}$ benutzt wird. Da auch das Duale zur Bemerkung a) gilt, erhält man allgemeiner folgende Aussage: Ist $U \subset Q$ und ist $Q$ selbstinjektiv, dann ist $U$ groß in $U^{* *}$ und $U^{* *}$ ist direkter Summand von $Q$. Ist $Q$ injektiv, dann ist folglich $U^{* *}$ injektiv und daher eine injektive Hïlle von $U$.

c) Hilfssatz. Ist A komplementiert und ist $f: A \rightarrow B$ ein Epimorphismus mit $\mathrm{Ke}(f:$ klein in $A$, dann ist auch $B$ komplementiert.

Beweis. Seien $V \subset B, U=f^{-1}(V)$ und $A=U+U^{\prime}$, dann zeigt man leicht, $\mathrm{daB} V^{\prime}=f\left(U^{\prime}\right)$.

Folgerung. Ist B ein Modul derart, daß jedes epimorphe Bild von $B$ eine projektive Hülle besitzt ${ }^{1)}$, dann ist $B$ komplementiert.

Beweis. Nach [1] ist die projektive Hülle von $B$ semi-perfekt also komplementiert und nach dem Hilfssatz gilt dies dann auch für $B$.

d) Ein Modul $M$ heißt endlich P-erzeugt, wenn $M$ epimorphes Bild einer endlichen direkten Summe von Kopien von $P$ ist. Sei $n$ eine natürliche $Z$ ahl und bezeichne $\mathscr{S}_{n}(P)$ die Klasse der Moduln, die epimorphe Bilder einer direkten Summe von $n$ Kopien von $P$ sind. Ein Modul $M$ heiße regulär, wenn jeder endlich erzeugte Untermodul von $M$ direkter Summand von $M$ ist (dies stimmt für Ringe mit dem Begriff regulär im Sinne von Neumann überein). Sei

$$
\delta=\delta(P, M)=\left\{f \mid f \in \operatorname{Hom}_{R}(P, M) \wedge B i(f) \text { klein in } M\right\} ;
$$

sei $S=\operatorname{Hom}_{R}(P, P)$, dann ist $H=\operatorname{Hom}_{R}(P, M) S$-Rechtsmodul und, wie leicht zu sehen, ist $\delta S$-Untermodul von $H$. Für $H$ und $\delta$ als $S$-Rechtsmoduln gilt dann der folgende Satz, dessen erster Teil auf $F$. Sandomierski zurückgeht (Spezialfall in [1]).

SaTZ.

1) Ist $P \mathbb{B}_{n}(P)$-projektiv und ist $M \in \mathbb{B}_{n}(P)$, dann gilt $\delta=\operatorname{Ra}\left(\operatorname{Hom}_{R}(P, M)\right)$.

2) Ist ausserdem $M$ komplementiert, dann ist $\operatorname{Hom}_{R}(P, M) / R a\left(\operatorname{Hom}_{R}(P, M)\right)$

1) Es dürte vielleicht $z$ weckmäßig sein, anders als in [1] bereits derartige Moduln (die also nicht notwendig projektiv sind) als semi-perfekt $z u$ bezeichnen. 
regulär.

Die Voraussetzungen sind z.B. alle erfüllt, wenn $P$ semi-perfekt und $M$ endlich $P$-erzeugt ist.

Dualisiert man diesen Satz, so erhält man eine Verallgemeinerung eines Satzes von Johnson und Wong [2].

Beim Beweis, der hier nicht ausgeführt werden soll, ist es zweckmäBig, vom Begriff eines quasi-regulären Untermoduls $U$ eines Moduls $M$ Gebrauch zu machen, den wir, da er (wie bei Ringen) selbständiges Interesse besitzt, noch erwähnen wollen. $U$ heiße quasi-regulärer Untermodul von $M$, wenn für jedes Erzeugendensystem $\left\{m_{i} \mid i \in \mathfrak{F}\right\}$ von $M$ und jede Teilmenge $\left\{u_{i} \mid i \in \mathfrak{F}\right\}$ von $U$ auch $\left\{m_{i}+u_{i} \mid i \in \mathfrak{F}\right\}$ Erzeugendensystem von $M$ ist. Es gilt dann, daB ein Untermodul genau dann quasi-regulär ist, wenn er klein ist und daß daher das Radikal $R a(M)$ von $M$ Summe aller quasi-regulären Untermoduln ist.

\section{LiTERATUR}

[1] Mares, E. A.: Semi-perfect modules. Math. Zeitschr. 82, 347-360 (1963).

[2] Johnson, R. E. and E. T. Wong: Self-injective rings. Can. Math. Bull. 2, 167-174 (1959).

München, Mathematisches Institut der Universität

Swarthmore College, Swarthmore, Pennsylvania 